Submarine Mass Movements and Their Consequences 
Advances in Natural and Technological Hazards Research

Volume 28

For other titles published in this series go to www.springer.com/series/6362 
David C. Mosher - R. Craig Shipp

Lorena Moscardelli • Jason D. Chaytor

Christopher D.P. Baxter - Homa J. Lee

Roger Urgeles

Editors

\section{Submarine Mass Movements and Their Consequences}

4th International Symposium

第 Springer 
David C. Mosher

Natural Resources Canada

Dartmouth, NS, Canada

dmosher@nrcan.gc.ca

Lorena Moscardelli

University of Texas at

Austin, TX, USA

lorena.moscardelli@beg.utexas.edu

Christopher D.P. Baxter

University of Rhode Island,

Narragansett, RI, USA

baxter@egr.uri.edu

Roger Urgeles

Institute of Marine Sciences,

Spanish National Research Council (CSIC)

urgeles@icm.csic.es
R. Craig Shipp

Shell International

Exploration and Production

Inc., Houston, TX, USA

Craig.Shipp@shell.com

Jason D. Chaytor

Woods Hole Oceanographic

Institution, Woods Hole,

MA, USA

jchaytor@usgs.gov

Homa J. Lee

United States Geological

Survey, Menlo Park, CA,

USA

hlee@usgs.gov

ISBN 978-90-481-3070-2

e-ISBN 978-90-481-3071-9

DOI 10.1007/978-90-481-3071-9

Springer Dordrecht Heidelberg London New York

Library of Congress Control Number: 2009933588

(c) Springer Science+Business Media B.V. 2010

No part of this work may be reproduced, stored in a retrieval system, or transmitted in any form or by any means, electronic, mechanical, photocopying, microfilming, recording or otherwise, without written permission from the Publisher, with the exception of any material supplied specifically for the purpose of being entered and executed on a computer system, for exclusive use by the purchaser of the work.

Printed on acid-free paper

Springer is part of Springer Science+Business Media (www.springer.com) 


\section{Dedication}

\section{William R. Normark Bruno Savoye 1943-2008 1959-2008}

This book, "Submarine Mass Movements and Their Consequences IV", is dedicated to the memory of two colleagues whose wide-ranging field studies did so much to advance our understanding of deep-water sedimentation systems: Drs. Bill Normark and Bruno Savoye. I had the good fortune to count them among my closest professional and personal friends. Both were sea-going scientists par excellence, both were warm and supportive individuals, who always had time for students, and both were modest about their own accomplishments.

Although many will remember Bill for his work on turbidites and submarine fans, he made important early contributions to our understanding of submarine mass movements. Large slides are a significant component of deep-water terrigenous basins and thus did not escape Bill's curiosity. In 1968, during his Ph.D. work, he acquired seabed imagery and sub-bottom profiles with the Scripps deeptow system over Ranger slide off Baja California. His 1974 and 1990 papers on this slide are classic Normark: meticulous use of multiple data sets and a clear mind as to what were the important issues. As Chief Scientist of the GLORIA cruise that mapped the seafloor around the Hawaiian islands, he was the first to appreciate the full extent of the flank-collapse debris avalanches, using the terms "prodigious" and "humongous" in the literature (and "giant" when reined in by editors).

Likewise, Bruno is remembered by many for his work on turbidite systems, but he too worked on submarine slides, particularly earlier in his career. The first paper that he and I worked on together described a slide near the wreck of the Titanic, where his colleagues at IFREMER had collected a magnificent survey with the next generation of deep-tow technology, the IFREMER SAR, but just missed the wreck. He is well known for his contributions on the 1979 Nice landslide, which triggered both a tsunami and a turbidity current. Bruno brought his enthusiasm and energy, together with his organisational and analytical skills as an engineer, to the science of turbidites and mass movements. He combined the technological expertise of IFREMER, industry funding and his own instinct of what was necessary to make significant scientific advances at sea.

Mass movements are an important part of the turbidite story. For Bill and Bruno, during much of their careers, mass movements were regarded as the predominant cause of turbidity currents. Both played an important role in challenging that dogma in recent years. Those who study mass movements from the comfort of the seismic work-station or by numerical modelling owe a lot to seagoing scientists like Bruno and Bill who laid the groundwork of where, why and how submarine mass-movements occur in the field. 


\section{Contents}

Submarine Mass Movements and Their Consequences

D.C. Mosher, L. Moscardelli, R.C. Shipp, J.D. Chaytor,

C.D.P. Baxter, H.J. Lee, and R. Urgeles

\section{SECTION I SUBMARINE MASS MOVEMENTS: TRIGGERS, MECHANICS, AND GEOTECHNICAL PROPERTIES}

Interplay Between Gas Hydrates and Submarine Slope Failure

J.L.H. Grozic

Advanced Dynamic Soil Testing - Introducing the New

Marum Dynamic Triaxial Testing Device

S. Kreiter, T. Moerz, M. Strasser, M. Lange, W. Schunn, B.F. Schlue,

D. Otto, and A. Kopf

Clustering of Geotechnical Properties of Marine

Sediments Through Self-Organizing Maps: An Example

from the Zakynthos Canyon-Valley System, Greece.

M.D. Ferentinou, T. Hasiotis, and M.G. Sakellariou

Identification of Shear Zones and Their Causal Mechanisms

Using a Combination of Cone Penetration Tests and Seismic

Data in the Eastern Niger Delta

S. Garziglia, N. Sultan, A. Cattaneo, S. Ker, B. Marsset, V. Riboulot, M. Voisset, J. Adamy, and S. Unterseh

Mass Wasting Dynamics at the Deeper Slope of the Ligurian Margin (Southern France)

A. Förster, V. Spieß, A. J. Kopf, and B. Dennielou 
Characterization of Micaceous Sand for Investigation

of a Subsea Mass Movement

T. Langford and S. Perkins

Estimating Drag Forces on Suspended and Laid-on-Seafloor

Pipelines Caused by Clay-Rich Submarine Debris Flow Impact

A. Zakeri

Experimental Investigation of Subaqueous Clay-Rich

Debris Flows, Turbidity Generation and Sediment Deposition

A. Zakeri, G. Si, J.D.G. Marr, and K. Høeg

The Kinematics of a Debris Avalanche on the Sumatra Margin

A.S. Bradshaw, D.R. Tappin, and D. Rugg

3D Numerical Modelling of Submerged and Coastal Landslide

Propagation.

P. Mazzanti, F. Bozzano, M.V. Avolio, V. Lupiano,

and S. Di Gregorio

Peculiar Morphologies of Subaqueous Landslide Deposits

and Their Relationship to Flow Dynamics

P. Mazzanti and F.V. De Blasio

Large Landslides on Passive Continental Margins:

Processes, Hypotheses and Outstanding Questions

D.G. Masson, R.B. Wynn, and P. J. Talling

Origin of Overpressure and Slope Failure

in the Ursa Region, Northern Gulf of Mexico

B. Dugan and J. Stigall

History of Pore Pressure Build Up and Slope Instability in Mud-Dominated Sediments of Ursa Basin, Gulf of Mexico

Continental Slope

R. Urgeles, J. Locat, D.E. Sawyer, P.B. Flemings, B. Dugan, and N.T.T. Binh

How Does Fluid Inflow Geometry Control Slope Destabilization?

I. Kock and K. Huhn

Geochemical Evidence for Groundwater-Charging of Slope Sediments: The Nice Airport 1979 Landslide and Tsunami Revisited

A.J. Kopf, S. Kasten, and J. Blees 
Modeling Slope Instability as Shear Rupture Propagation

in a Saturated Porous Medium

R.C. Viesca and J.R. Rice

\section{SECTION II SUBMARINE MASS MOVEMENTS: CASE STUDIES AND HAZARD ASSESSMENT}

Submarine Mass Transport Within Monterey Canyon:

Benthic Disturbance Controls on the Distribution

of Chemosynthetic Biological Communities

C.K. Paull, B. Schlining, W. Ussler III, E. Lundsten, J.P. Barry,

D.W. Caress, J.E. Johnson, and M. McGann

Multi-direction Flow in a Mass-Transport Deposit,

Santos Basin, Offshore Brazil

L.B. Ashabranner, E.K. Tripsanas, and R.C. Shipp

Small-Scale Insights into Seismic-Scale Slumps:

A Comparison of Slump Features from the Waitemata Basin,

New Zealand, and the Møre Basin, Off-Shore Norway....

S. Bull and J. Cartwright

The Block Composite Submarine Landslide,

Southern New England Slope, U.S.A.: A Morphological Analysis

J. Locat, U.S. ten Brink, and J.D. Chaytor

Post-Megaslide Slope Stability North of Svalbard, Arctic Ocean

D. Winkelmann, W.H. Geissler, R. Stein, and F. Niessen

Geomorphology of the Talismán Slide (Western slope

of Hatton Bank, NE Atlantic Ocean)

M. Sayago-Gil, D. Long, L.-M. Fernández-Salas, K. Hitchen,

N. López-González, V. Díaz-del-Río, and P. Durán-Muñoz

Investigations on the Peach 4 Debrite, a Late Pleistocene

Mass Movement on the Northwest British Continental Margin

M. Owen, S. Day, D. Long, and M. Maslin

Redistribution of Sediments by Submarine Landslides on the Eastern Nankai Accretionary Prism.

K. Kawamura, T. Kanamatsu, M. Kinoshita, S. Saito, T. Shibata,

K. Fujino, A. Misawa, and K.C. Burmeister

Mass Wasting at the Easternmost Cyprus Arc, Off Syria,

Eastern Mediterranean

E. Tahchi, R. Urgeles, C. Hübscher, and J. Benkhelil 
Risk Analysis for Hurricane-Wave Induced

Submarine Mudslides ....

M.C. Nodine, R.B. Gilbert, J.Y. Cheon, S.G. Wright, and E.G. Ward

GIS-Based Assessment of Submarine Mudflow Hazard

Offshore of the Mississippi Delta, Gulf of Mexico

C. Hitchcock, R. Givler, M. Angell, and J. Hooper

Spatial Analysis of Shallow Slope Instability Incorporating

an Engineering Geological Ground Model

B. Mackenzie, J. Hooper, and D. Rushton

Estimating the Empirical Probability of Submarine Landslide

Occurrence

E.L. Geist and T. Parsons

Constraining Geohazards to the Past: Impact Assessment of Submarine Mass Movements on Seabed Developments

S. Thomas, J. Hooper, and M. Clare

Evaluating Gas-Generated Pore Pressure with Seismic

Reflection Data in a Landslide-Prone Area: An Example

from Finneidfjord, Norway

E.C. Morgan, M. Vanneste, O. Longva, I. Lecomte, B. McAdoo, and L. Baise

Historic and Paleo-Submarine Landslide Deposits Imaged

Beneath Port Valdez, Alaska: Implications for Tsunami

Generation in a Glacial Fiord

H.F. Ryan, H.J. Lee, P.J. Haeussler,

C.R. Alexander, and R.E. Kayen

Multibeam Bathymetry Investigations of Mass Movements

in Lake Le Bourget (NW Alps, France) Using a Portable Platform

G. Ledoux, P. Lajeunesse, E. Chapron, and G. St-Onge

Morphodynamic and Slope Instability Observations

at Wabush Lake, Labrador

D. Turmel, J. Locat, G. Cauchon-Voyer, C. Lavoie, P. Simpkin,

G. Parker, and P. Lauzière

Climate-Induced Turbidity Current Activity in NW-African

Canyon Systems.

R. Henrich, T.J.J. Hanebuth, Y. Cherubini, S. Krastel, R. Pierau, and C. Zühlsdorff 


\section{SECTION III SUBMARINE MASS MOVEMENTS IN MARGIN CONSTRUCTION AND ECONOMIC SIGNIFICANCE}

Investigating the Timing, Processes and Deposits of One of the World's Largest Submarine Gravity Flows:

The 'Bed 5 Event' Off Northwest Africa

R.B. Wynn, P.J. Talling, D.G. Masson, C.J. Stevenson, B.T. Cronin, and T.P. Le Bas

MTCs of the Brazos-Trinity Slope System; Thoughts on the Sequence Stratigraphy of MTCs and Their Possible Roles in Shaping Hydrocarbon Traps

R.T. Beaubouef and V. Abreu

\section{Southeast Australia: A Cenozoic Continental Margin}

Dominated by Mass Transport

R. Boyd, J. Keene, T. Hubble, J. Gardner, K. Glenn, K. Ruming,

N. Exon, and the crews of Southern Surveyor 10/2006 and 12/2008

A Database on Submarine Landslides of the Mediterranean Sea 503

A. Camerlenghi, R. Urgeles, and L. Fantoni

Submarine Landslides Along the Algerian Margin:

A Review of Their Occurrence and Potential Link with Tectonic Structures....

A. Cattaneo, N. Babonneau, G. Dan, J. Déverchère, A. Domzig,

V. Gaullier, B. Lepillier, B.M. de Lépinay, A. Nouguès,

P. Strzerzynski, N. Sultan, and K. Yelles

Mass-Transport Deposits on the Algerian Margin (Algiers Area): Morphology, Lithology and Sedimentary Processes

G. Dan, N. Sultan, A. Cattaneo, J. Déverchère, and K. Yelles

Detailed Analysis of a Submarine Landslide (SAR-27)

in the Deep Basin Offshore Algiers (Western Mediterranean)

A. Nouguès, N. Sultan, A. Cattaneo, G. Dan, K. Yelles, and PRISME team

3D Seismic Interpretation of Mass Transport Deposits:

Implications for Basin Analysis and Geohazard Evaluation

J. Frey-Martínez

Slope Instability on the French Guiana Transform Margin from Swath-Bathymetry and $3.5 \mathrm{kHz}$ Echograms

V. Gaullier, L. Loncke, L. Droz, C. Basile, A. Maillard, M. Patriat, W.R. Roest, B. Loubrieu, L. Folens, and F. Carol 
Megaslides in the Foz do Amazonas Basin, Brazilian

Equatorial Margin

C.G. Silva, E. Araújo, A.T. Reis, R. Perovano, C. Gorini,

B.C. Vendeville, and N. Albuquerque

Detached and Shelf-Attached Mass Transport Complexes

on the Magdalena Deepwater Fan

G.A. Romero-Otero, R.M. Slatt, and C. Pirmez

Character, Distribution and Timing of Latest Quaternary

Mass-Transport Deposits in Texas-Louisiana Intraslope

Basins Based on High-Resolution $(3.5 \mathrm{kHz})$ Seismic Facies

and Piston Cores.

607

H.C. Olson and J.E. Damuth

Upper Cretaceous Mass Transport Systems Above

the Wyandot Formation Chalk, Offshore Nova Scotia

B.M. Smith, M.E. Deptuck, and K.L. Kendell

The Significance of Mass-Transport Deposits for the Evolution of a Proglacial Continental Slope

T.J. Huppertz, D.J.W. Piper, D.C. Mosher, and K. Jenner

\section{SECTION IV SUBMARINE MASS MOVEMENTS AND TSUNAMIS}

Middle to Late Miocene Slope Failure and the Generation of a Regional Unconformity Beneath the Western Scotian

Slope, Eastern Canada.

D.C. Campbell and D.C. Mosher

Mass Transport Deposits on the Southwestern Newfoundland Slope

M.K. Giles, D.C. Mosher, D.J.W. Piper, and G.D. Wach

Mass Transport Events and Their Tsunami Hazard

D.R. Tappin

Hydrodynamic Modeling of Tsunamis Generated by Submarine

Landslides: Generation, Propagation, and Shoreline Impact.

P. Lynett

Calculations of Tsunamis from Submarine Landslides

G. Gisler, R.P. Weaver, and M. Gittings

Experiments on Tsunamis Generated by 3D Granular Landslides 705

F. Mohammed and H.M. Fritz 
Distal Turbidites and Tsunamigenic Landslides of Stromboli

Volcano (Aeolian Islands, Italy)

A. Di Roberto, M. Rosi, A. Bertagnini, M.P. Marani, and F. Gamberi

Tsunamigenic Risks Associated with Mass Transport

Complexes in Offshore Trinidad and Venezuela

L. Moscardelli, M. Hornbach, and L. Wood

Distribution and Tsunamigenic Potential of Submarine

Landslides in the Gulf of Mexico

J.D. Chaytor, D.C. Twichell, P. Lynett, and E.L. Geist

A Study of the Tsunami Effects of Two Landslides in the St. Lawrence Estuary

R. Poncet, C. Campbell, F. Dias, J. Locat, and D. Mosher

The Pliocene Shelburne Mass-Movement and Consequent

Tsunami, Western Scotian Slope

D.C. Mosher, Z. Xu, and J. Shimeld

Author Index.

Subject Index

CD-ROM enclosed 


\section{Contributors}

\section{Abreu}

ExxonMobil, Houston, TX, USA

\section{J. Adamy}

TOTAL, Paris La Défense, France

\section{N. Albuquerque}

Universidade Federal Fluminense, Departamento de Geologia, Av. Gen. Milton

Tavares de Souza, s.n., Niterói, RJ, CEP: 24210-346, Brazil

\section{C.R. Alexander}

Skidaway Institute of Oceanography, Savannah, GA, USA

\section{Angell}

Fugro (William Lettis \& Associates, Inc.), 1777 Botelho Drive, Suite 262, Walnut Creek, CA 94598, USA

\section{E. Araújo}

Universidade Federal Fluminense, Departamento de Geologia, Av. Gen. Milton Tavares de Souza, s.n., Niterói, RJ, CEP: 24210-346, Brazil

\section{L.B. Ashabranner}

Shell International E \& P Inc. 200 N. Dairy Ashford, Houston, TX 77079, USA

\section{M.V. Avolio}

Department of Mathematics, University of Calabria, Arcavacata, 87036 Rende (CS), Italy

\section{N. Babonneau}

UBO-IUEM, CNRS UMR6538 Domaines Océaniques, 29280 Plouzané, France

\section{Baise}

Tufts University, Dept of Civil and Envir Eng, 200 College Ave, Anderson Hall, Rm 113, Medford, MA 02155, USA

\section{J.P. Barry}

Monterey Bay Aquarium Research Institute, Moss Landing, CA 95039-9644, USA 


\section{Basile}

Laboratoire de Géodynamique des Chaînes Alpines, UMR-CNRS 5025,

Observatoire des Sciences de l'Univers de Grenoble, Université Joseph Fourier, Maison des Géosciences, 1381 rue de la Piscine, 38400 St. Martin d'Hères, France

\section{R.T. Beaubouef}

Hess Corporation, Houston, TX, USA

\section{A. Bertagnini}

Istituto Nazionale di Geofisica e Vulcanologia, Sezione di Pisa, Via della Faggiola, 32 - 56126 Pisa, Italy

\section{J. Benkhelil}

IMAGES, University of Perpignan Via Domitia, Perpignan 66860 Cedex, France

\section{N.T.T. Binh}

Durham University, Department of Earth Sciences, Science Labs, Durham DH1 3LE, UK

\section{J. Blees}

Alfred Wegener Institute for Polar and Marine Research, 27570 Bremerhaven, Germany

Present address: University of Basel, 4056 Basel, Switzerland

\section{R. Boyd}

ConocoPhillips, Houston and University of Newcastle, Australia

\section{F. Bozzano}

Dipartimento di Scienze della Terra, Università di Roma "Sapienza", P.le Aldo Moro 5, 00185 Roma, Italy

\section{A.S. Bradshaw}

Merrimack College, North Andover, MA 01845, USA

\section{S. Bull}

Cardiff University, 3D Lab, School of Earth, Ocean and Planetary Sciences, Main Building, Park Place Cardiff CF10 3YE, UK

\section{K.C. Burmeister}

University of the Pacific, Department of Geosciences, 3601, Pacific Avenue, Stockton, California 95211, USA

\section{A. Camerlenghi}

ICREA, Istitució Catalana de Recerca i Estudis Avançats, Barcelona, Spain; Departament d'Estratigrafia, Paleontologia i Geociències Marines, Facultat de Geologia, Universitat de Barcelona, C/Martí i Franquès, s/n, E-08028 Barcelona, Spain

\section{D.C. Campbell}

Geological Survey of Canada, Natural Resources Canada, Bedford Institute of Oceanography, 1 Challenger Dr., Dartmouth, NS, Canada, B2Y 4A2; Department of Earth Sciences, Dalhousie University, Halifax, Nova Scotia, Canada, B3H 4R2 


\section{F. Carol}

Laboratoire IMAGES - E.A. 4218, Université de Perpignan Via Domitia, 52 Avenue Paul Alduy, 66860 Perpignan Cedex, France

\section{J. Cartwright}

Cardiff University, 3D Lab, School of Earth, Ocean and Planetary Sciences, Main Building, Park Place Cardiff CF10 3YE, UK

\section{A. Cattaneo}

IFREMER, Géosciences Marines, Laboratoire Environnements Sédimentaires, Plouzané, France

\section{G. Cauchon-Voyer}

Laboratoire d'études sur les risques naturels,

Département de géologie et de génie géologique, Université Laval, Québec, Qc, Canada, G1K 7P4

\section{E. Chapron}

Université d'Orléans, CNRS/INSU, Université François Rabelais-Tours, Institut des Sciences de la Terre d'Orléans (ISTO)-UMR 6113, 1A rue de la Férollerie, F-45071 Orléans cedex 2, France

\section{D.W. Caress}

Monterey Bay Aquarium Research Institute, Moss Landing, CA 95039-9644, USA

\section{J.D. Chaytor}

United States Geological Survey, Woods Hole Science Center, 384 Woods Hole Rd., Woods Hole, MA 02543, USA

\section{J.Y. Cheon}

The University of Texas at Austin, Department of Civil, Architectural and Environmental Engineering, Geotechnical Engineering Program, 1 University Station, C1792, Austin, Texas 78712-0280, USA

\section{Clare}

Fugro GeoConsulting Limited, Engineering Geology and Geohazards Team, Fugro House, Hithercroft Road, Wallingford, Oxfordshire, OX10 9RB, UK

\section{B.T. Cronin}

Deep Marine, 9 North Square, Footdee, Aberdeen, AB11 5DX, UK

\section{J.E. Damuth}

Department of Earth and Environmental Sciences, University of Texas, 500 Yates St., 107 Geoscience Bldg., Arlington, TX 76019, USA

\section{G. Dan}

Université de Brest; CNRS, UMR 6538 Domaines Océaniques; Institut Universitaire Européen de la Mer, Place Copernic, 29280 Plouzané, France; FUGRO FRANCE S.A.S, 27 Rue des Peupliers, 92752, Nanterre, France; IFREMER, Géosciences Marines, Laboratoire Environnements Sédimentaires, Plouzané, France 


\section{S. Day}

Aon Benfield UCL Hazard Research Centre, Department of Earth Sciences, University College London, Gower Street, London WC1E 6BT, UK

\section{F.V. De Blasio}

Department of Geosciences, University of Oslo/ P.O. Box 1047 Blindern, N-0316 Oslo, Norway \& International Centre of Geohazards, P.O. Box 3930 Ulleval Stadion, N-0806 Oslo, Norway

\section{B. Dennielou}

IFREMER - French Research Institute for Exploitation of the Sea,

Département des Géosciences Marines, PO box 70, 29280

Plouzané Cédex, France

\section{M.E. Deptuck}

Canada-Nova Scotia Offshore Petroleum Board, 1791 Barrington St, Halifax, NS, B2Y 4A2, B.M. de Lépinay UMR6526 CNRS Géosciences Azur, Valbonne, France

\section{J. Déverchère}

UBO-IUEM, CNRS UMR6538 Domaines Océaniques, 29280 Plouzané, France

\section{F. Dias}

Centre de Mathématiques et de Leurs Applications, Ecole Normale Supérieure de Cachan and CNRS, France

\section{Díaz-del-Río}

Instituto Español de Oceanografia, Centro Oceanográfico de Málaga, Puerto Pesquero, s/n. Apdo. 285, 29640 Fuengirola (Málaga), Spain

\section{S. Di Gregorio}

Department of Mathematics, University of Calabria, Arcavacata, 87036 Rende (CS), Italy

\section{A. Di Roberto}

Istituto Nazionale di Geofisica e Vulcanologia, Sezione di Pisa, Via della Faggiola, 32 - 56126 Pisa, Italy

\section{A. Domzig}

Midland Valley Exploration, Glasgow, UK L.Droz UMR-CNRS 6538, IUEM, Place Nicolas Copernic, 29280 Plouzané, France

\section{B. Dugan}

Rice University, Department of Earth Science, 6100 Main Street, Houston, Texas 77005, USA

\section{P. Durán-Muñoz}

Instituto Español de Oceanografía, Centro Oceanográfi co de Vigo, Cabo Estay-Canido, Apdo. 1552, 36200 Vigo (Pontevedra), Spain

\section{N. Exon}

Department of Earth and Marine Sciences, Australian National University, ACT 0200, Australia 


\section{Fantoni}

Dipartimento di Scienze della Terra, Università di Modena

e Reggio Emilia, Via S. Eufemia, 19, 41100 Modena, Italy

\section{M.D. Ferentinou}

School of Rural and Surveying Engineering, Laboratory of Structural Mechanics, National Technical University of Athens, 15780 Zografou, Greece

\section{L.-M. Fernández-Salas}

Instituto Español de Oceanografia, Centro Oceanográfico de Málaga, Puerto Pesquero, s/n. Apdo. 285, 29640, Fuengirola (Málaga), Spain

\section{P.B. Flemings}

University of Texas at Austin, Jackson School of Geosciences,

1 University Station C1100, Austin, TX 78712-0254, USA

\section{Folens}

Laboratoire Géosystèmes, UMR 8157, Université des Sciences et Technologies de Lille 1, Cité Scientifique, Bâtiment SN5, 59655 Villeneuve d'Ascq Cedex, France

\section{A. Förster}

MARUM- Center for Marine Environmental Sciences, University of Bremen, Leobener Straße, PO box 330440, 28334 Bremen, Germany

\section{J. Frey-Martínez}

Repsol-YPF, Repsol Oil Operations Libya (Akakus), Airport Road,

Tripoli, Libya

\section{H.M. Fritz}

School of Civil and Environmental Engineering, Georgia Institute of Technology, 210 Technology Circle, Savannah, GA 31407, USA

\section{K. Fujino}

Kyushu University, 10-1 Hakozaki, Higashi-ku, Fukuoka

812-8581, Japan

\section{F. Gamberi}

Istituto di Scienze Marine - CNR, Sede di Geologia Marina di Bologna, Via Gobetti 101 - 40129 Bologna, Italy

\section{J. Gardner}

CCOM, University of New Hampshire, USA

\section{S. Garziglia}

IFREMER, BP 70, 29280 Plouzané Cedex, France

\section{Gaullier}

Laboratoire IMAGES - E.A. 4218, Université de Perpignan Via Domitia, 52 Avenue Paul Alduy, 66860 Perpignan Cedex, France

\section{W.H. Geissler}

Alfred Wegener Institute for Polar and Marine Research, Bremerhaven, Germany 


\section{Eric L. Geist}

U.S. Geological Survey, 345 Middlefield Road, MS 999.

Menlo Park, CA 94025, USA

\section{R.B. Gilbert}

The University of Texas at Austin, Department of Civil, Architectural and Environmental Engineering, Geotechnical Engineering Program, 1 University Station, C1792, Austin, Texas 78712-0280, USA

\section{M.K. Giles}

Department of Earth Sciences, Dalhousie University, Halifax, N.S., Canada, B3H 4J1

\section{G. Gisler}

Physics of Geological Processes, University of Oslo, PO Box 1048 Blindern, 0316 Oslo, Norway

\section{Gittings}

Science Applications International, 3900 North Ocean Dr, \#11A, Lauderdale by the Sea, Florida 33308, USA

\section{R. Givler}

Fugro (William Lettis \& Associates, Inc.), 1777 Botelho Drive, Suite 262, Walnut Creek, CA 94598, USA

\section{K. Glenn}

Geoscience Australia, Canberra, Australia

\section{Gorini}

Université Pierre \& Marie Curie, Paris VI,.Laboratoire de Tectonique et Modélisation des Bassins Sédimentaires, UMR 7072, Place Jussieu, Case 117 tour 46-00, 75252 Paris, Cedex 05, France

\section{Jocelyn L.H. Grozic}

Department of Civil Engineering, University of Calgary, ENF 262, 2500 University Dr NW, Calgary, Alberta, Canada, T2N 1N4

\section{P.J. Haeussler}

U.S. Geological Survey, Anchorage, AK, USA

\section{T. Hasiotis}

Department of Marine Sciences, University of the Aegean, University Hill, 81100 Mytilene, Lesvos, Greece

\section{R. Henrich}

University of Bremen, Faculty of Geosciences and MARUM, PO Box 330 440, 28334 Bremen, Germany

\section{K. Hitchen}

British Geological Survey, West Mains Road, Edinburgh, EH9 3LA, UK 


\section{Hitchcock}

Fugro (William Lettis \& Associates, Inc.), 1777 Botelho Drive, Suite 262, Walnut Creek, CA 94598, USA

\section{K. Høeg}

Department of Geosciences, University of Oslo, P.O. Box 1047 Blindern, NO-0316 Oslo, Norway; International Centre for Geohazards (ICG), Sognsveien 72, 0855, Oslo, Norway

\section{J. Hooper}

Fugro GeoConsulting Limited, Engineering Geology and Geohazards Team, Fugro House, Hithercroft Road, Wallingford, Oxfordshire, OX10 9RB, UK

\section{Hornbach}

Institute for Geophysics, Jackson School of Geosciences, The University of Texas at Austin, TX, USA

\section{T. Hubble}

University of Sydney, Australia

\section{Hübscher}

Institut für Geophysik, Universität Hamburg, Bundesstraße 55, 20146 Hamburg, Germany

\section{K. Huhn}

MARUM - Center for Marine Environmental Sciences, University of Bremen, PO box 330 440, D-28334, Bremen, Germany

\section{T.J. Huppertz}

University of Bremen, Faculty of Geosciences, FB 5 Klagenfurter Strasse 28359

Bremen, Germany

\section{K. Jenner}

Geological Survey of Canada, Natural Resources Canada, Bedford Institute of Oceanography, 1 Challenger Drive, Dartmouth, Nova Scotia, B2Y 4A2, Canada

\section{J.E. Johnson}

University of New Hampshire, Durham, NH 03824, USA

\section{T. Kanamatsu}

IFREE1, Japan Agency for Marine Science and Technology, 2-15 Natsushima-cho, Yokosuka, Kanagawa 237-0061, Japan

\section{S. Kasten}

Alfred Wegener Institute for Polar and Marine Research, 27570 Bremerhaven, Germany

\section{K. Kawamura}

Fukada Geological Institute, 2-13-12 Hon-Komagome, Bunkyo, Tokyo 113-0021, Japan 


\section{R.E. Kayen}

U.S. Geological Survey, 345 Middlefield Rd., MS 999,

Menlo Park, CA, USA

\section{J. Keene}

University of Sydney, Australia

\section{K.L. Kendell}

Canada-Nova Scotia Offshore Petroleum Board, 1791 Barrington St, Halifax, NS, Canada B2Y 4A2

\section{S. Ker}

IFREMER, BP 70, 29280 Plouzané Cedex, France

\section{Kinoshita}

IFREE1, Japan Agency for Marine Science and Technology, 2-15 Natsushima-cho, Yokosuka, Kanagawa 237-0061, Japan

\section{Kock}

MARUM - Center for Marine Environmental Sciences, University of Bremen, PO box 330 440, D-28334, Bremen, Germany

\section{A. Kopf}

MARUM - Center for Marine Environmental Sciences

and Faculty of Geosciences, University Bremen, Leobener Str., 28334 Bremen, Germany

\section{S. Kreiter}

MARUM - Center for Marine Environmental Sciences and Faculty

of Geosciences, University Bremen, Leobener Str. 28334 Bremen, Germany

\section{P. Lajeunesse}

Université Laval, Centre d'études nordiques \& Département de géographie, Québec, QC, Canada G1V 0A6

\section{Lange}

MARUM - Center for Marine Environmental Sciences

and Faculty of Geosciences, University Bremen, Leobener Str., MARUM, 28334 Bremen, Germany

\section{T. Langford}

NGI, P.O. Box 3930 Ullevål Stadion, NO-0806 Oslo, Norway

\section{P. Lauzière}

Iron Ore Company of Canada, Environment Department, P.O. Box 10000, Labrador City, NL, Canada A2V 2L8

\section{Lavoie}

Department of Geosciences, Hamilton College, Clinton, New York, 13323 USA

\section{T.P. Le Bas}

National Oceanography Centre, Southampton, European Way, Southampton, SO14 3ZH, UK 


\section{Lecomte}

NORSAR/ICG, P.O. Box 53, N-2027 Kjeller, Norway

\section{G. Ledoux}

Université Laval, Centre d'études nordiques \& Département

de géographie, Québec, QC, Canada G1V 0A6

\section{H.J. Lee}

U.S. Geological Survey, 345 Middlefield Rd., MS 999, Menlo Park, CA, USA

\section{B. Lepillier}

Ifremer, GM-LES, BP 70, 29280 Plouzané, France pres. add. TOTAL, Stavanger, Norway

\section{J. Locat}

Université Laval, Dept. of Geology and Geological Engineering, Québec, Canada G1K 7P4

\section{Loncke}

Laboratoire IMAGES - E.A. 4218, Université de Perpignan Via Domitia, 52 Avenue Paul Alduy, 66860 Perpignan Cedex, France

\section{Long}

British Geological Survey, West Mains Road, Edinburgh, EH9 3LA, UK

O. Longva

NGU/ICG, 7491 Trondheim, Leiv Eirikssons vei 39, Trondheim, Norway

\section{N. López-González}

Instituto Español de Oceanografia, Centro Oceanográfico de Málaga, Puerto Pesquero, s/n. Apdo. 285, 29640 Fuengirola (Málaga), Spain

\section{B. Loubrieu}

IFREMER, Géosciences Marines, BP70, 29280 Plouzané, France

\section{E. Lundsten}

Monterey Bay Aquarium Research Institute, Moss Landing, CA 95039-9644, USA

\section{Lupiano}

Department of Earth Sciences, University of Calabria, Arcavacata, 87036 Rende (CS), Italy

\section{P. Lynett}

Texas A\&M University, College Station, TX 77843, USA

\section{B. Mackenzie}

Fugro GeoConsulting Limited, Fugro House, Hithercroft Road, Wallingford, Oxfordshire, OX10 9RB, UK

\section{A. Maillard}

LMTG, Université UPS (SVT-OMP), 14 Avenue Edouard Belin, 31400 Toulouse, France 


\section{J.D.G. Marr}

National Center for Earth-Surface Dynamics, St. Anthony Falls Laboratory, Minneapolis, MN, USA

\section{M.P. Marani}

Istituto di Scienze Marine - CNR, Sede di Geologia Marina di Bologna,

Via Gobetti 101 - 40129 Bologna, Italy

\section{B. Marsset}

IFREMER, BP 70, 29280 Plouzané Cedex, France

\section{Maslin}

Environmental Change Research Centre, Department of Geography,

College London, Gower Street, London WC1E 6BT, UK

\section{D.G. Masson}

National Oceanography Centre, Southampton, European Way, Southampton, SO14 3ZH, UK

\section{P. Mazzanti}

Dipartimento di Scienze della Terra, Università di Roma "Sapienza", P.le Aldo Moro 5, 00185 Roma, Italy

\section{B. McAdoo}

Vassar College, Dept of Earth Sci and Geog, Poughkeepsie, NY 12604, USA

\section{McGann}

U.S. Geological Survey, Menlo Park, CA 94025, USA

\section{F. Mohammed}

School of Civil and Environmental Engineering, Georgia Institute of Technology, 210 Technology Circle, Savannah, GA 31407, USA

\section{Moscardelli}

Bureau of Economic Geology, Jackson School of Geosciences, The University of Texas at Austin, TX, USA

\section{E.C. Morgan}

Tufts University, Dept of Civil and Envir Eng, 200 College Ave, Anderson Hall, Rm 113, Medford MA 02155, USA

\section{D.C. Mosher}

Geological Survey of Canada, Natural Resources Canada

Bedford Institute of Oceanography, 1 Challenger Drive, Dartmouth, Nova Scotia, B2Y 4A2, Canada

\section{A. Misawa}

Tokai University; 3-20-1 Orido, Shimizu, Shizuoka 424-8610, Japan

\section{T. Moerz}

MARUM - Center for Marine Environmental Sciences and Faculty of Geosciences, University Bremen, Leobener Str., 28334 Bremen, Germany 


\section{F. Niessen}

Alfred Wegener Institute for Polar and Marine Research, Bremerhaven, Germany

\section{M.C. Nodine}

GEI Consultants, Inc.,1790 38th St. \#103, Boulder, CO 80302, USA

\section{A. Nouguès \\ Institut Polytechnique LaSalle Beauvais, Département Géosciences, Beauvais, France; FUGRO FRANCE S.A.S., Nanterre, France}

\section{H.C. Olson}

Institute for Geophysics, Jackson School of Geosciences, The University of Texas, 10100 Burnet Rd., Bldg. 196 (ROC), Austin, TX 78758, USA

\section{Otto}

MARUM - Center for Marine Environmental Sciences

and Faculty of Geosciences, University Bremen, Leobener Str., 28334 Bremen, Germany

\section{Owen}

Environmental Change Research Centre, Department of Geography,

College London, Gower Street, London WC1E 6BT, UK

\section{G. Parker}

Ven Te Chow Hydrosystems Lab, Department of Civil Engineering, College of Engineering, University of Illinois at Urbana-Champaign, IL 61802, USA

\section{T. Parsons}

U.S. Geological Survey, 345 Middlefield Road, MS 999. Menlo Park, CA 94025, USA

\section{Patriat}

IFREMER, Géosciences Marines, BP70, 29280 Plouzané, France

\section{C.K. Paull}

Monterey Bay Aquarium Research Institute, Moss Landing, CA 95039-9644, USA

\section{S. Perkins}

Montana State University, 205 Cobleigh Hall, Bozeman MT 59717, USA

\section{R. Perovano}

Universidade Federal Fluminense, Departamento de Geologia, Av. Gen. Milton Tavares de Souza, s.n., Niterói, RJ, CEP: 24210-346, Brazil

\section{D.J.W. Piper}

Geological Survey of Canada, Natural Resources Canada Bedford Institute of Oceanography, 1 Challenger Drive, Dartmouth,

Nova Scotia, B2Y 4A2, Canada

\section{Pirmez}

Shell International E and P., Houston, TX, USA 


\section{R. Poncet}

Centre de Mathématiques et de Leurs Applications, Ecole Normale Supérieure de Cachan and CNRS, France

\section{A.T. Reis}

Universidade do Estado do Rio de Janeiro, Faculdade de Oceanografia, Rua São Francisco Xavier, 524, $4^{\circ}$ Andar, bl E, Rio de Janeiro/RJ.

CEP: 20.550-013, Brazil

\section{Riboulot}

IFREMER, BP 70, 29280 Plouzané Cedex, France

\section{J.R. Rice}

School of Engineering and Applied Sciences (also, for JRR,

Department of Earth and Planetary Sciences), Harvard University,

29 Oxford St., Cambridge, MA 02138, USA

\section{W.R. Roest}

IFREMER, Géosciences Marines, BP70, 29280 Plouzané, France

\section{G.A. Romero-Otero}

Conoco Phillips School of Geology and Geophysics, The University of Oklahoma, Norman, OK, USA

\section{Rosi}

University of Pisa, Dipartimento di Scienze della Terra, Via S. Maria, 53 - 56126 Pisa, Italy

\section{Rugg}

University of Texas at Austin, Austin, Texas, USA

\section{K. Ruming}

NSW Department of Primary Industries, Maitland, Australia

\section{Rushton}

Fugro GeoConsulting Limited, Fugro House, Hithercroft Road, Wallingford, Oxfordshire, OX10 9RB, UK

\section{H.F. Ryan}

U.S. Geological Survey, 345 Middlefield Rd., MS 999, Menlo Park, CA, USA

\section{S. Saito}

IFREE1, Japan Agency for Marine Science and Technology, 2-15 Natsushima-cho, Yokosuka, Kanagawa 237-0061, Japan

\section{M.G. Sakellariou}

School of Rural and Surveying Engineering, Laboratory of Structural Mechanics, National Technical University of Athens, 15780 Zografou, Greece

\section{D.E. Sawyer}

University of Texas at Austin, Jackson School of Geosciences, 1 University Station C1100, Austin, TX 78712-0254, USA 


\section{Sayago-Gil}

Instituto Español de Oceanografia, Centro Oceanográfico de Málaga, Puerto

Pesquero, s/n. Apdo. 285, 29640 Fuengirola (Málaga), Spain

B. Schlining

Monterey Bay Aquarium Research Institute, Moss Landing, CA 95039-9644, USA

\section{B.F. Schlue}

ARCADIS Consult GmbH, Europaplatz, 3, 64293 Darmstadt, Germany

\section{W. Schunn}

MARUM - Center for Marine Environmental Sciences and Faculty of

Geosciences, University, Bremen, Leobener Str., 28334 Bremen, Germany

\section{T. Shibata}

Kochi University, 2-5-1 Akebono-cho, Kochi 780-8520, Japan

\section{J. Shimeld}

Geological Survey of Canada, Natural Resources Canada Bedford, Institute of Oceanography, 1 Challenger Dr., Dartmouth, NS, Canada, B2Y 4A2

\section{R.C. Shipp}

Shell International E \& P Inc. 200 N. Dairy Ashford, Houston, TX 77079, USA

G. Si

Department of Geosciences, University of Oslo, P.O. Box 1047 Blindern, NO-0316 Oslo, Norway

\section{C.G. Silva}

Universidade Federal Fluminense, Departamento de Geologia, Av. Gen. Milton

Tavares de Souza, s.n., Niterói, RJ, CEP: 24210-346, Brazil

\section{P. Simpkin}

IKB Technologies Limited, 1220 Hammonds Plains Road, Bedford, Nova Scotia, Canada B4B 1B4

\section{R.M. Slatt}

Conoco Phillips School of Geology and Geophysics, The University of Oklahoma, Norman, OK, USA

\section{B.M. Smith}

Canada-Nova Scotia Offshore Petroleum Board, 1791 Barrington St, Halifax, NS, Canada B2Y 4A2

\section{Spieß}

MARUM- Center for Marine Environmental Sciences, University of Bremen, Leobener Straße, PO box 330440, 28334 Bremen, Germany

\section{R. Stein}

Alfred Wegener Institute for Polar and Marine Research, Bremerhaven, Germany 


\section{C.J. Stevenson}

National Oceanography Centre, Southampton, European Way, Southampton, SO14 3ZH, UK

\section{J. Stigall}

Department of Earth Science, Rice University, 6100 Main Street, MS 126,

Houston, TX 77005, USA

\section{G. St-Onge}

Université du Québec à Rimouski, Institut des sciences de la mer de Rimouski (ISMER), Rimouski, QC, Canada, G5L 3A1

\section{Strasser}

MARUM - Center for Marine Environmental Sciences and Faculty of Geosciences, University, Bremen, Leobener Str., 28334 Bremen, Germany

\section{P. Strzerzynski}

UBO-IUEM, CNRS UMR6538 Domaines Océaniques, 29280 Plouzané, France

\section{N. Sultan}

IFREMER, Géosciences Marines, Laboratoire Environnements Sédimentaires, Plouzané, France

\section{E. Tahchi}

Departament d'Estratigrafi a, Paleontologia i Geociències Marines, Facultat de Geologia, Universitat de Barcelona, Martí i Franquès, s/n, 08028 Barcelona, Catalonia, Spain

\section{P.J. Talling}

National Oceanography Centre, Southampton, European Way, Southampton, SO14 3ZH, UK

\section{D.R Tappin}

British Geological Survey, Nottingham, NG12 5GG, UK

\section{U.S. ten Brink}

United States Geological Survey, Woods Hole Science Center, 384 Woods Hole Rd., Woods Hole, MA 02543, USA

\section{S. Thomas}

Fugro GeoConsulting Limited, Engineering Geology and Geohazards Team, Fugro House, Hithercroft Road, Wallingford, Oxfordshire, OX10 9RB, UK

\section{E.K. Tripsanas}

Shell International E \& P Inc. 200 N. Dairy Ashford, Houston, TX 77079, USA

\section{Turmel}

Laboratoire d'études sur les risques naturels, Département de géologie et de génie géologique, Université Laval, Québec, Canada G1K 7P4

\section{D.C. Twichell}

U.S. Geological Survey, Woods Hole, MA 02543, USA 


\section{S. Unterseh}

TOTAL, Paris La Défense, France

\section{R. Urgeles}

Departament d'Estratigrafi a, Paleontologia i Geociències Marines, Facultat de Geologia, Universitat de Barcelona, Martí i Franquès, s/n, 08028 Barcelona, Catalonia, Spain

\section{W. Ussler III}

Monterey Bay Aquarium Research Institute, Moss Landing, CA 95039-9644, USA

\section{Vanneste}

NGI/ICG, P.O. Box 3930, Ullevål Stadion, N-0806 Oslo, Norway

\section{B.C. Vendeville}

Université de Lille1, 4 UMR 8157 Géosystèmes, Bat. SN5, USTL, 59655, Villeneuve d'Ascq, Cedex - France

\section{R. C. Viesca}

School of Engineering and Applied Sciences (also, for JRR, Department of Earth and Planetary Sciences), Harvard University, 29 Oxford St., Cambridge, MA 02138, USA

\section{Voisset}

IFREMER, BP 70, 29280 Plouzané Cedex, France

\section{G.D. Wach}

Department of Earth Sciences, Dalhousie University, Halifax, N.S., Canada B3H 4J1

\section{E.G. Ward}

Offshore Technology Research Center, Texas A\&M University, 1200 Mariner Drive, College, Station, TX 77845, USA

\section{R.P. Weaver}

Los Alamos National Laboratory, MS T086, Los Alamos, New Mexico 87545, USA

\section{Winkelmann}

Leibniz Institute for Marine Science (IFM-GEOMAR), Kiel, Germany

\section{Wood}

Bureau of Economic Geology, Jackson School of Geosciences, The University of Texas at Austin, TX, USA

\section{S.G. Wright}

The University of Texas at Austin, Department of Civil, Architectural and Environmental, Engineering, Geotechnical Engineering Program, 1 University Station, C1792, Austin, Texas, 78712-0280, USA 


\section{R.B. Wynn}

National Oceanography Centre, Southampton, European Way, Southampton, SO14 3ZH, UK

\section{Z. Xu}

Canadian Hydrographic Service, Fisheries and Oceans Canada, Maurice-LaMontagne Laboratory PO, Box 1000, Mont-Joli, Quebec, G5H 3Z4 Canada

\section{K. Yelles}

CRAAG, Centre de Recherche en Astronomie, Astrophysique et Géophysique, Bouzaréah, Algiers, Algeria

\section{A. Zakeri}

Geotechnical Engineering Group, C-CORE, St. John's, Newfoundland, Canada; International Centre for Geohazards (ICG), Sognsveien 72, 0855, Oslo, Norway 